\title{
Understanding the relationship between pain and emotion in idiopathic headaches
}

\author{
G. Bussone · L. Grazzi
}

(C) Springer-Verlag Italia 2013

\begin{abstract}
The aim of this paper is to review hypotheses regarding pain mechanisms in headache and relationships between headache pain and the brain's emotional network. There is evidence that chronic pain in idiopathic headaches is, in part, an emotional response induced by alterations in the homeostasis of the interoceptive system-a system that integrates nociceptive information with the emotional network (mediating emotional awareness). These findings suggest that idiopathic headaches are probably due to both an altered pain matrix on the one hand, and an altered affective-cognitive state on the other.
\end{abstract}

Keywords Pain · Emotion · Headache

Knowledge of the pathophysiology of headache has increased markedly in recent years. We have moved from the idea that peripheral mechanisms are responsibleextracranial (muscle contraction, vascular dilatation) or intracranial (vascular innervation of the dura) - to the notion that the central nervous system (CNS), and particular the pain modulating circuits, are primarily involved in the genesis of idiopathic headaches like migraine [1].

According to this view, the initiating event for head pain occurs in the vicinity of the peripheral terminals of primary afferent dural nociceptors. The initiating event leads to depolarization of the nociceptor terminal, generating action potentials that are propagated to synapses on second-order neurons of the trigemino-cervical complex [2]. From there,

G. Bussone $(\bowtie) \cdot$ L. Grazzi

Headache Unit, Department of Clinical Neuroscience,

C. Besta Neurological Institute and Foundation, Via Celoria,

11, 20133 Milan, Italy

e-mail: bussone.g@istituto-besta.it the signal is relayed via well-known pathways through the thalamus to reach cortical areas concerned with the perception of head pain [3]. In fact there are two pain pathways: the neospinothalamic tract and the paleospinothalamic tract.

The neospinothalamic tract is an almost direct periphery-spinal cord-brain pathway, typically conveying acute pain sensations that undergo minimal modulation before reaching the cortex, and providing sensory-discriminative information ("how and where"). However, this kind of perception, defined "nociception", is not yet pain. To feel pain, it is necessary that messages transmitted via the paleospinothalamic system arrive at the cerebral cortex. The paleospinothalamic system is concerned with affective aspects of pain, and signals undergo modulation at several points en route to the cortex to change how pain is eventually perceived. Both the neo- and paleo-spinothalamic systems interact with cortical association areas [4].

However, assuming that only these pain transmission pathways are involved, idiopathic headaches such as migraine have characteristics that are difficult to explain.

For example, it is well established that headache frequency and intensity can be influenced-and perhaps headaches can be triggered-by psychological factors such as stress, attention, expectation, relaxation-based therapies and suggestion [5]. In addition, during pain-free periods, migraineur brains are hyper-excitable [6], and while experiencing headache, responses to various stimuli are abnormal. Thus, in many migraineurs, sensory stimuli such as light, odours and noise can exacerbate the headache, and sleep onset can abort severe headaches. This cerebral hyper-excitability might be due to altered balance between excitatory (e.g. glutamate) and inhibitory (e.g. GABA) neurotransmitters [7]. If this were the case, it would provide a rationale for the use of the so-called neuromodulator drugs in migraine prophylaxis, since these substances act 
by changing the balance between glutamate and GABA in the brain [8].

Finally, notwithstanding earlier beliefs that dilated cranial blood vessels, plasma serotonin, and cortical spreading depression precede or accompany certain headache types [9], most idiopathic headaches (including migraine without aura and tension type headache) are not associated with a distinguishing biomarker or with objective changes in peripheral tissues.

It seems important, therefore, to turn our attention to the overlap between the brain systems underlying emotion and those concerned with pain and pain modulation. Clinicians concerned with treating headache are well aware that stressing stimuli play a role in triggering a migraine or a cluster period. This experience suggests that the cortical and subcortical circuits that regulate emotion have a significant influence on the regulation of pain. And we need to understand the mechanisms by which this interaction takes place to improve our understanding of idiopathic headaches.

It is clear that headache is not a neuropathic pain. The IASP definition of pain is "an unpleasant sensory and emotional experience associated with actual or potential tissue damage, or described in terms of such damage" [10] and characteristically includes:

(a) Pain and sensory symptoms that persist beyond healing,

(b) Variable presence of sensory neurological signs manifesting as negative or positive sensory phenomena,

(c) Variable presence of other neurological signs, including motor signs (manifesting as negative and positive motor phenomena) and autonomic signs.

None of these characteristics apply to migraine pain. So we should consider the possibility that idiopathic headaches are not purely pain conditions, but instead might represent a physical response to functional alterations in the CNS structures that underlie emotion [11]. And this is more likely to be the cases in patients with chronic recurring headache [12].

We know that various cortical and subcortical structures are critical for the expression and experience of emotional states: limbic system-related structures such as the anterior cingulate cortex, frontal medial cortex, anterior temporal cortex, amygdala, hippocampus, and related thalamic and hypothalamic nuclei. Furthermore, functional magnetic imaging (fMRI) data obtained during migraine attacks demonstrate that the limbic system is activated. The insula and amygdala-limbic system structures-influence the so-called pain matrix, via the hypothalamus and, in particular, are involved in the inhibition of nociception [13].
There is also evidence that the brainstem control of pain is altered in migraine, in particular, activation of the periaqueductal grey matter (PAG)-implicated in defence reactions, and together with the hypothalamus in the regulation of the autonomic system-has been demonstrated during a migraine attack [14].

The basal ganglia are also implicated in migraine [15]. They are known to be involved in the modulation of pain signals and the modulation of higher cortical function, in particular, via the frontostriatal circuits.

Persons with major depression have been reported to have reduced volume of the orbitofrontal cortex, and, as is well-known, persons with chronic migraine are often depressed [16]. Furthermore, a PET study [17] has shown orbitofrontal cortex hypometabolism both in patients with chronic analgesic-overuse headache evolving from episodic migraine, and in patients with drug dependence. Similarly, an fMRI study carried out by our group [18] showed that female patients with chronic migraine with medication overuse (CMwMO) had hypoactivity, compared to controls, in some areas of the lateral pain pathway, primary somatosensory cortex (S1) and posterior parietal cortex (PPC), following noxious mechanical stimuli. These hypoactive areas returned to normal activity within 6 months of medication withdrawal, suggesting that hypoactivity may have been induced by the overuse of symptomatic medications. Another possibility, however, is that it may have been due to chronic cortical downregulation that stopped the pain impulses arriving at the cortex (activity-dependent plasticity arising from the excessive pain inputs that occur during migraine attacks). The reversibility of this phenomenon following medication withdrawal, accompanied by decreased incidence of headache attacks, suggests that no major neuron loss occurs [18].

To test whether lateral pain pathway hypoactivity in CMwMO patients was due to medication overuse or to altered chronic pain processing, we studied a group of chronic migraine patients (CM) not overusing medication. We submitted them to the same noxious mechanical stimuli as in the previous study. We found that, while CMwMO patients showed hypoactivity of some areas of the lateral pain pathway, CM patients without medication overuse did not show any hypoactivity in these regions (personal data). It, therefore, seems likely that hypoactivity in CMwMO is due to medication overuse and not to chronic pain input. The medication overuse might also be responsible for lowering the pain threshold and amplifying responses to migraine triggers, leading to increased frequency of headache attacks. The fact that CM patients without medication overuse do not seem to have neuroplastic changes in the lateral pain pathway suggests that different factors are 
involved in headache chronification in these two groups of patients.

From on these findings it is reasonable to speculate that chronic pain is an emotional response arising from an alteration in the homeostasis of the interoceptive systemthe system that integrates nociceptive information with emotional awareness [19].

Migraine is today considered an illness of the brain. It is characterized by multiple alterations in various cortical and subcortical pathways, and these alterations may arise as a plastic response to repeated pain episodes (in those with chronic migraine), as a result of genetic predisposition, or as a consequence of both. We would go further: recent findings, reviewed briefly above, suggest that the pain of primary migraine is a sign of homeostatic imbalance in the interoceptive system, and that it leads to adaptive responses such as withdrawal, motor quiescence, sympathetic inhibition and lethargy. By contrast, motor agitation (characteristic of $\mathrm{CH}$ ) would be a fight or flight reaction mediated by the interoceptive system. A consequence of this hypothesis is that personality traits influence not only whether chronic migraine will develop but also whether treatment will be successful. In particular, if treatment is not integrated with biopsychological approaches, it will have a higher probability of failure.

Feeling pain seems to be an essential component in the dynamic of emotions and hence the genesis and driving of behaviour. For chronic migraineurs, pain would be just one component (a major one) of the behavioural response to their condition. This perspective suggests new directions for research-greatly aided by the still-developing functional imaging technologies - that will not only generate new insights into the pathophysiology of primary headaches, but also clarify the relationship between the neurolimbic circuits and pain modulation.

Acknowledgments I thank Don Ward for helpful in the preparation of this article.

Conflict of interest I certify that there is no actual or potential conflict of interest in relation to this article.

\section{References}

1. Olesen J, Burstein R, Ashina M, Tfelt-Hansen P (2009) Origin of pain in migraine: evidence for peripheral sensitisation. Lancet Neurol 8:679-690
2. Hargreaves R, Shepheard SL (1999) Pathophysiology of migraine: new insights. Can J Neurol Sci 26(3):S12-S19

3. Fields HL (1997) Pain modulation and headache. In: Goadsby PJ, Silberstein SD (eds) Headache: blue books of practical neurology, vol 17. Butterworth-Heinemann, Boston

4. Schiff M (1858) Lehrbuch der physiologie des Menschen: I. muskel und Nervenphysiologie. Schauenburg M, Lahr

5. Welch KMA (1987) Migraine a biobehavioral disorder. Arch Neurol 44:323-327

6. Welch KMA, D'Andrea G, Tepley N et al (1990) The concept of migraine as a state of central neuronal hyperexcitability. Neurol Clin 8:817-828

7. D'Andrea G, Perini F, Terrazzino S, Nordera GP (2004) Contributions of biochemistry to the pathogenesis of primary headaches. Neurol Sci 3(3):589-592

8. Diener HC, Bussone G, Van Oene JC, Lahaye M, Schwalen S, Goadsby PJ, TOPMAT-MIG-201 (TOP-CHROME) Study Group (2007) Topiramate reduces headache days in chronic migraine: a randomized, double-blind, placebo-controlled study. Cephalalgia $27: 814-823$

9. Zhang X, Levy D, Kainz V et al (2011) Activation of central trigeminovascular neurons by cortical spreading depression. Ann Neurol 69:855-865

10. Merskey H, Bogduk N (eds) (1994) Classification of chronic pain, 2nd edn. IASP Task force on Taxonomy. IASP Press, Seattle

11. Craig AD (2003) A new view of pain as homeostatic emotion. Trends Neurosci 26(6):303-307

12. Bussone G, Grazzi L, Panerai AE (2012) Pain, emotion, headache. Headache 52(S2):98-101.

13. Holland P, Goadsby PJ (2007) The hypothalamic orexinergic system: pain and primary headaches. Headache 47(6):951-962

14. Weiller C, May A, Limmroth V et al (1995) Brain stem activation in spontaneous human migraine attacks. Nat Med 1:658-660

15. Biagianti B, Grazzi L, Gambini O et al (2012) Orbitofrontal dysfunction and medication overuse in patient with migraine. Headache 52:1511-1519

16. Torelli P, Lambru G, Manzoni GC (2006) Psychiatric comorbidity and headache: clinical and therapeutical aspects. Neurol Sci 27(2):S73-S76

17. Fumal A, Laureys S, Di Clemente L et al (2006) Orbitofrontal cortex involvement in chronic analgesic-overuse headache evolving from episodic migraine. Brain 129(Pt 2):543-550

18. Grazzi L, Chiapparini L, Ferraro S et al (2010) Chronic migraine with medication overuse pre-post withdrawal: clinical results and fMRI correlations. Headache 50:998-1004

19. Craig AD (2002) How do you feel? Interoception: the sense of the physiological condition of the body. Nat Rev Neurosci 3(8): 655-666 